\title{
De la domesticación de la investigación cualitativa al reto de reinventarse*
}

\author{
José J. Barba, Raúl A. Barba-Martín, ${ }^{2}$ Gustavo González-Calvo ${ }^{3}$ \\ Universidad de Valladolid (España)
}

Recibido: enero 29 de 2015 - Revisado: febrero 17 de 2015 - Aceptado: mayo 15 de 2015

Referencia formato APA: Barba, J. J., Barba-Martin, R., \& González-Calvo, G. (2015). De la domesticación de la investigación cualitativa al reto de reinventarse. Revista Cientifica Guillermo de Ockham, 13(1), 51-58.

\section{Resumen}

La investigación cualitativa trata de un modelo basado en conseguir transformaciones que fomenten la justicia social. En ella, el investigador actúa en comunidad con los participantes, quienes son los dueños del problema y como tales deben ser el eje de la investigación y centra los procesos en conseguir resultados que transformen las prácticas sociales. Sin embargo, las presiones con las que los investigadores se encuentran desde las administraciones y las empresas que financian las investigaciones, hacen que la investigación cualitativa entre en un conflicto entre su verdadera función y conseguir finalidades objetivas y extrapolables, lo que desvirtúa la investigación cualitativa. Frente a esta problemática, es importante tener presente las características de la investigación cualitativa para reconducirla hacia la finalidad transformadora con la que se creó.

Palabras clave: investigación cualitativa, cultura de las auditorías, evidencias, participación, cuestionamientos del investigador

\section{From the qualitative research domestication to the challenge of reinventing}

\section{Abstract}

Qualitative research is a model based on getting transformations that promote social justice. For this, the researcher acts with the participants, who are the owners of the problem and as such, should be the core of the research, focusing the processes in getting results which transform social practices. However, the pressures that researchers have from the administrations and companies that finance the researches make qualitative research encounter a conflict between its true function and obtaining objective and extrapolated purposes, thereby distorting qualitative research. Against this problem it is important to consider the characteristics of qualitative research to redirect it to the transformation purpose for which it was created.

Keywords: Qualitative research, auditory culture, evidences, inquiry research

\footnotetext{
* Este artículo se deriva del proyecto I+D Las competencias docentes en la formación inicial del profesorado de educación física. Convocatoria de noviembre de 2013 del Programa Estatal de Investigación, Desarrollo e Innovación Orientada a los Retos de la Sociedad, en el marco del Plan Estatal de Investigación Científica y Técnica y de Innovación 2013-2016. Referencia: EDU 2013-42024-R.

1. Doctor en Educación, Facultad de Educación de Segovia de la Universidad de Valladolid; jjbarba@pdg.uva.es; Campus María Zambrano, Universidad de Valladolid, Plaza Alto de los Leones, 1; 40004 Segovia (Espańa). E-mail: jjbarba@pdg.uva.es

2. Estudiante de doctorado en el Centro Transdisciplinar de Educación de la Universidad de Valladolid (CETIE-UVa). E-mail: raulbarba13@gmail. com

3. Doctor en Educación, Facultad de Educación de Palencia de la Universidad de Valladolid. E-mail: gustavo.gonzalez@uva.es
} 


\section{Domesticação de pesquisa qualitativa para o desafio de reinventar}

\section{Resumo}

A pesquisa qualitativa é baseada na obtenção de transformaçôes que promovam o modelo de justiça social. Nele, as obras pesquisador em participantes da comunidade são os donos do problema e como tal deve ser o foco da pesquisa, com foco na obtenção de resultados de processos que transformam as práticas sociais . No entanto, as pressóes que os pesquisadores são da pesquisa administraçóes e empresas de fundo faz com que a pesquisa qualitativa em um conflito entre sua verdadeira função e obter finalidades objetivas e extrapolados, distorcendo assim a pesquisa qualitativa. Confrontado com este problema é importante considerar as características da pesquisa qualitativa para redirecioná-lo para o propósito de transformação para o qual ele foi criado.

Palavras chave: Ensino superior; pesquisa narrativa; solidez metodológica; movimento epistêmico-político

\section{Introducción}

El modelo universitario se está transformando y como investigadores cualitativos comprometidos con el cambio social debemos replantearnos dónde nos encontramos. Estos tiempos se caracterizan por la imposición de un modelo neoliberal en la sociedad y, por ende, en la investigación, si bien nos enfrentamos con un modelo muy sutil que poco a poco nos va calando dentro. Apenas existen guerras evidentes por colonizar el pensamiento y dominar los territorios, sobre todo Europa y Norteamérica se encuentran en una situación de paz bélica. Pero esto no implica que la dominación no se pueda ejercer de otras formas. Baudrillard (1981) hace referencia a la seducción como una forma de obtener el poder. En la misma línea, Fromm (2006) afirma que la autoridad puede ser anónima, basada en la seducción sin el uso evidente de la fuerza. Parecen lejanos los ańos ochenta y el lema tacherista "No hay alternativa”, en los que se comenzó a fomentar que las cosas son como son y por mucho que deseemos cambiarlas no es posible hacerlo.

Esta falta de esperanza ha ido calando en el discurso social y nos lleva a asumir colectivamente la imposibilidad de modificar la realidad en la que vivimos, conllevando así un pensamiento único (Lather, 2012). En este nuevo campo de juego, se nos plantea una serie de dilemas: ¿qué potencial tiene la investigación cualitativa si-como afirman Denzin y Lincoln (2011) - se encuentra orientada a transformar el mundo? ¿Cuál es el espacio de juego en el que la investigación cualitativa se está desarrollando? ¿Qué opciones tenemos de actuación y de investigación de cara al futuro? A lo largo del artículo abordaremos estas cuestiones y los retos a los que nos enfrentamos como investigadores cualitativos.

\section{Definiendo sutilmente el concepto de investigación}

Consideramos que las transformaciones sociales de las que se ha visto contagiada la investigación, han expulsado conceptualmente a la investigación cualitativa del espacio donde se desarrolla la investigación. Pero esta exclusión está limitada a la forma de entender la investigación cualitativa y no a las técnicas que permiten desarrollarla; es decir, se siguen considerando válidas las formas, pero no los intereses que las construyeron. En este sentido, Flick (2015) expone que el desarrollo de investigaciones cualitativas no va aparejado a un crecimiento en las cuestiones epistemológicas. Por contra, en la investigación cualitativa las cuestiones sobre el paradigma son más importantes que las metodológicas (Guba, Lynham \& Lincoln, 2011), por lo que eliminar la reflexión sobre nuestra forma de investigar provoca un proceso de domesticación por el sistema que se desarrolla a través de tres cuestionamientos desde el campo de las prácticas políticas.

El primer cuestionamiento es el uso del término evidencia, como el resultado al que toda investigación ha de llegar y que ha de demostrar su validez. El diccionario de la RAE lo define como una "certeza de la que no se puede dudar"; algo difícil de conseguir en la investigación con personas. Si bien un objeto se comporta siempre igual con determinadas variables, en el caso de las personas no; cada una tiene su forma de interiorizar el mundo y de actuar ante él, por lo que las actuaciones no son previsibles. De hecho, si retomamos a Bruner (2012) nos encontramos con que el ser humano es a la vez herencia genética que lo predispone a hacer cosas, pero es su voluntad la que determina. Es decir, si un nińo decide no aprender, independientemente de su coeficiente intelectual y de 
las evidencias logradas mediante la investigación, no aprenderá. Este hecho provocará que se dude de la certeza conseguida por la investigación, por lo que no será una evidencia. Sin embargo, respecto de la columna de un puente, si se han hecho bien los cálculos, sí tendremos la evidencia de que no se caerá.

Aun conociendo que es prácticamente imposible obtener evidencias en el sentido de "certeza de la que no se puede dudar", nos encontramos que en determinados espacios políticos hay que definir el concepto de evidencia -o logro derivado de la investigación- $y$, en consecuencia, los mejores métodos para conseguirla y los estándares para evaluarla (Denzin, 2009; Morse, 2006). El establecimiento de la evidencia corresponde a resultados esperados a partir de un criterio externo, estableciendo con ello estándares e indicadores ajenos a los colectivos que investigan y a las propias lógicas de la investigación. Denzin y Lincoln (2011), consideran que el nacimiento de la investigación cualitativa fue una forma de oposición de los investigadores al positivismo, propiciando de esta manera una forma diferente de entender la investigación. En la actualidad, esa diferencia se ha borrado por la cultura de las evidencias y por servir a los interés productivos (Rodríguez-Romero, 2015), lo que aleja a la investigación cualitativa de sus perspectivas iniciales que, según Lather (2006), se aproximaban al campo de la justicia social y de la equidad. De forma subyacente, la política de las evidencias está ligada con una forma de ética de mercado (Denzin, 2011; Shamir, 2008).

Una vez definida la evidencia parece oportuno decidir cómo llegar a ella; es decir, hay que definir qué es una investigación. En el caso de las ciencias sociales, hemos de tener claro que no nos encontramos ante una realidad construida por los participantes y sus interacciones. Pero si ante la falta de una realidad objetiva se ha definido un logro derivado de la investigación mesurable, ya no tenemos que orientar nuestra acción a conocer la realidad, sino a buscar evidencias de lo que pretendemos. En ese sentido se apoya la utilización de datos cuantitativos con muestras aleatorias que supuestamente permiten generalizaciones (Rodríguez Romero, 2015). Así, se fomentan estudios en los que lo importante es la predicción y no la comprensión (Denzin, 2009). Además, la investigación cualitativa no se guía por un proceso lineal y objetivado al ser una realidad multimetodológica que se aplica en función del contexto y de los criterios del investigador (Barba, 2013).

El modelo de investigación predictiva -alejado de lo cualitativo- se impone a través de formas sutiles, como los criterios para financiar las investigaciones, la valora- ción positiva del desarrollo de patentes y la creación de empresas dedicadas a explotar esas patentes -spin-off(Barba, González Calvo \& Barba-Martín, 2014). Para esta cuestión, el potencial de la investigación cualitativa es menor que el de la cuantitativa, pero además implica alejarse de su propia epistemología y quedar al servicio de las demandas externas. Esto es un contrapunto con los orígenes donde los investigadores cualitativos crearon sus propios estándares con sus propios criterios (Lather, 2006). Recordemos lo ya expuesto cuando Flick (2015) reconoce que el desarrollo de la investigación cualitativa no está siendo parejo al desarrollo epistemológico. Podría parecer que los investigadores cualitativos estamos perdiendo la capacidad de definir nuestro campo y nuestros fines.

Como tercer aspecto de domesticación nos encontramos con la cultura de las auditorías, que vela por que las normas se cumplan desde una realidad con múltiples aristas que nos afectan como investigadores y académicos. Las auditorías borran las fronteras entre las disciplinas y las metodologías (Jemielniak \& Greenwood, 2015) buscando con ello una única forma de evaluar la generación de conocimiento, sin tener en cuenta la complejidad que requieren las ciencias sociales. Estas dinámicas van colapsando poco a poco los espacios de los investigadores y terminan siendo asumidas como lo "normal" y como una realidad en la que no hay alternativa y llega a ser naturalizada por los propios investigadores. En este sentido, Bourdieu (1997) expone que las auditorías, en pos de la objetividad y del pensamiento racional y científico, tienen como finalidad perpetuar el status quo dominante.

Las auditorías están presentes de una forma poliédrica. Se dan diferentes tipos como:

- La necesidad de que las investigaciones sean aprobadas por un comité previo (Giroux \& Tremblay, 2004)

- El establecimiento de un escalafón entre profesores (Sparkes, 2013)

- Las políticas editoriales de las revistas que definen los documentos elaborados para la revisión de manuscritos (Denzin, 2010a)

- La concepción de que los procesos de revisión por pares forman parte de una auditoría (Giroux \& Tremblay, 2004), olvidando así que estas revistas están auditadas por bases de datos y estas, a su vez, por las entidades y gobiernos financiadores que deciden el valor que otorga a cada una y en consecuencia a las revistas que se encuentran incluidas 
- La concesión de proyectos de investigación con determinados requisitos que imponen las entidades financiadoras y dan poco margen a la toma de decisiones por el investigador (Barba et al., 2014)

- La creación de agencias de evaluación del profesorado universitario que, principalmente en función de las publicaciones y sus índices de impacto, limitan el acceso a figuras del profesorado (Barba et al., 2014).

\section{La investigación sin ideología (evidente)}

El triduo de procesos descritos en el apartado anterior: elección de evidencias, cómo llegar a ellas y los mecanismos de evaluación, es asumido como parte del proceso investigador, en el entendido de que es la realidad que nos ha tocado vivir. No generar un debate sobre el propio sistema de investigación, hace que se imponga la cultura de las evidencias y de las auditorías como una realidad dada a la que no nos podemos enfrentar. Pero no podemos olvidar que uno de los logros de la investigación cualitativa ha sido poner de manifiesto que la objetividad y la neutralidad son imposibles en las ciencias sociales. Freire (1968/2007) consideraba que, como persona, hay que considerarse participante de una realidad, para desde ahí contraponer las diferentes perspectivas. Esta es una visión ética no adoctrinadora, lo que en sí implica no pasar por el mundo de forma neutral, como ya expone en su visión de la investigación. Pero la ruptura con la neutralidad y la objetividad se encuentra en la esencia de la investigación cualitativa, puesto que en su concepción profunda la vincula con la justicia social y la transformación (Denzin, 2010b; Denzin \& Lincoln, 2011; Flick, 2015; Lather, 2006). Además, la elección de los objetivos de una investigación ya es de por sí un acto en el que se encuentra presente la ideología del investigador (Vasilachis de Gialdino, 2009). Sobre esta base, si lo delegamos en el financiador del proyecto, será su concepción del mundo la que se encuentre implícita.

En la actualidad es difícil retomar la idea de Kuhn (1962/2004) respecto de los paradigmas cuantitativos y cualitativos como dos formas diferentes y opuestas de entender la investigación. Si bien hasta el momento se ha ido alternando la primacía entre ellos (Rodríguez-Romero, 2015), actualmente la búsqueda de las evidencias está borrando la frontera entre paradigmas con el desarrollo de los métodos mixtos. En un primer momento solo se planteaba la utilización de métodos cualitativos y cuantitativos en la misma investigación, y el debate sobre su paradigma quedaba abierto (Creswell, 2011). No somos contrarios a la complementariedad de métodos, pero nos cuestionamos qué visión tiene el investigador que utiliza métodos mixtos sobre la realidad, si busca predecir, transformar la sociedad o buscar evidencias. Esto deja el paradigma en función del investigador y si este no se lo plantea, puede asumir la evidencia como una realidad dada sin escapatoria.

Consideramos que se puede encontrar la lógica de la investigación actual recurriendo a otras formas de clasificar la investigación; en este caso, distinguiendo entre investigación básica y aplicada (la básica busca incrementar el conocimiento teórico y la aplicada tiene como fin generar conocimiento que se pueda transferir a la calle). Para Jemielniak (2006) uno de los problemas de la universidad, sobre todo en el caso de las ciencias sociales, proviene de la consideración de su investigación como poco práctica y poco útil, si bien esto está cambiando gracias a la producción de investigaciones rápidas y a los ingresos económicos. Esto parece ir en contra de una de las funciones fundamentales de la universidad, a saber, ser una institución crítica (Greenwood, 2012), que está siendo domesticada a través de su relación con el mercado laboral (Barnett, 2001). Dentro de esta lógica, es conveniente analizar cómo los resultados de la Investigación + Desarrollo $(\mathrm{I}+\mathrm{D})$ universitaria no solo son medidos en función de su productividad científica, sino también en función de las patentes generadas o las empresas dedicadas a explotar esas patentes -spin-off- (Barba et al., 2014).

\section{Dando fuerza a la investigación cualitativa}

Ante lo expuesto, nos encontramos con una investigación cualitativa que ha perdido parte de su sentido primigenio, aunque como conjunto de técnicas todavía sigue teniendo fuerza en las ciencias sociales. Consideramos que, como investigadores cualitativos, nos debemos replantear nuestros principios para reencontrarnos en nuestra práctica y hacer frente a la cultura de las evidencias y la cultura de las auditorías. Para ello, proponemos las preguntas que siguen a continuación.

\section{Para qué investigamos}

Esta pregunta trasciende los debates meramente técnicos que plantea la investigación en términos de cómo y qué. En nuestro caso, nos planteamos las finalidades que ha de tener la investigación, porque esta dice mucho acerca de cómo se usan los instrumentos a nuestro cargo y no cómo los instrumentos nos pueden servir para buscar evidencias. Diferentes autores (Denzin \& Lincoln, 2011; Potter, 1996; Villasante \& Montañés, 2000), consideran que la investigación cualitativa puede ser utilizada como 
un elemento de dominación sobre los grupos que investigados. No podemos olvidar que la investigación cualitativa consigue un conocimiento profundo de una realidad concreta, que puede ser utilizado para fines perversos respecto del propio grupo.

En este sentido, consideramos que el regreso a los orígenes de la investigación cualitativa, debe conllevar un ponerse al lado del débil en lo que es un acto de justicia social (Lather, 2006). La investigación cualitativa ha de recuperar el compromiso de ser un instrumento que denuncie las injusticias y transforme las sociedades. En este sentido, Greenwood (2000) considera que una concepción ética de la investigación implica que todas las personas que participan se encuentren integradas en los procesos como protagonistas de la investigación. De esta forma, podemos reconsiderar la investigación como un retorno a la justicia social basada en la democracia y en el abandono de otras concepciones, incluida la paternalista.

\section{Quién da sentido a nuestra investigación}

En este sentido, las personas implicadas se han ido transformando de sujetos a participantes. Últimamente, ha tomado fuerza la palabra inglesa stakeholder, grupo o individuo que puede verse afectado por el logro del objetivo pretendido (Freeman, 1984) y en esta dirección cabe considerar que los participantes de una investigación están interesados en un problema, pero no podemos olvidar que las empresas financiadoras y los gobiernos que patrocinan las investigaciones manejan sus propios intereses respecto de estas. Lincoln (2012), afirma que el Consejo Nacional de Investigación de Estados Unidos considera que los principales consumidores de investigación educativa son los propios investigadores y los políticos, idea que conlleva la crítica de que también debería estar incluida la comunidad como parte interesada. Consideramos que el peligro de una expresión como "interesados en el problema" radica en que puede afectar a tantos colectivos que corremos el riesgo de dejar a alguno importante fuera.

Entendemos que el compromiso de la investigación cualitativa no es la presentación del informe final, sino que su resultado mejore la justicia social. En este sentido, Greenwood (2000) propone el cambio del concepto "interesados en el problema" por "dueños del problema". Aunque pueda perecer un pequeńo matiz conceptual, la diferencia entre ambos es enorme puesto que la noción propuesta por Greenwood nos lleva a entender que aunque puede haber diferentes colectivos interesados, uno es el realmente afectado y vive objetivamente las consecuencias de forma directa. El uso de esta concepción permite acer- carnos a la idea de justicia social propia de la investigación cualitativa e integrar las demandas de los otros colectivos interesados sin perder el norte.

\section{Qué investigamos}

Una de las principales cuestiones en investigación es fijar un objetivo; es decir, qué queremos conocer. Entendemos este como un tercer momento de la investigación después de considerar los fines que nos mueven y los grupos que queremos que se beneficien. Creemos necesarias estas reflexiones previas para combinarlo con las presiones de los financiadores y de los distintos comités en el enunciado de los objetivos. En este sentido, la matización es un trabajo arduo por los miles de matices que se entrelazan; como por ejemplo, en el caso del verbo inicial. No es lo mismo conocer, lo cual presupone una idealizada neutralidad, que comprender, lo que da un papel activo a la persona que redacta el informe. Cada pequeño matiz puede encerrar de una forma sutil muchos matices, que hacen que de una forma imperceptible, se decante la investigación de diferentes maneras.

Si la investigación cualitativa tiene como fin la lucha por la justicia social, no lo puede perseguir de espaldas a los dueńos del problema. Entendemos que la investigación conlleva un compromiso ético entre participantes e investigador, que en ocasiones acarrea romper barreras como en el caso de las autoetnografías, en las que el investigador-participante actúa como etnógrafo y se expone a sí mismo y a su contexto de forma crítica (Ellis, 2004; Spry, 2001). Sin embargo, el compromiso ético puede ser roto, porque la investigación cualitativa tiene el potencial de ser usada para dominar o controlar grupos a partir del conocimiento que se obtiene de investigarlos, como ya hemos tratado. Para evitar que los intereses de los dueños del problema estén presentes en los objetivos de la investigación, es necesario que participen de su elaboración o al menos que participen en un diálogo ético sobre el posicionamiento de la investigación (Carducci, Pasque, Kuntz, \& Contreras-McGavin, 2013). Además, esto cuenta con la ventaja de que si el proceso investigador da respuesta a los objetivos planteados, quedarán resueltas las cuestiones tanto del grupo de investigadores como del colectivo que participa en la investigación, teniendo de esta manera una transferencia real.

\section{Quién ha de conocer los resultados}

Una de las principales cuestiones en investigación es qué se hace con los resultados, aunque quizás pueda parecer una cuestión obvia en estos tiempos cuando nos 
encontramos inmersos en el dilema de "publicar o morir" y las investigaciones cobran sentido en función del impacto de la revista en la que se publican los resultados. No podemos engańarnos respecto de la transferencia de resultados a la comunidad, puesto que la importancia de la revista en la que se publica el artículo, poco o nada tiene que ver con el impacto social de la investigación. Es el ejemplo de la temática de la educación (Hargreaves \& Shirley, 2012; Rivera García, 2012), en el que el logro no se vincula con la justicia social, sino con el número de citas en un grupo restringido de publicaciones. Pero este sistema también se muestra vulnerable al estar sujeto a un comercio perverso (Lawrence, 2008; Quindós, 2009).

Hemos abordado la vinculación entre la investigación y los interesados en el problema, en cuyos resultados reside el mayor peligro. Si volvemos al concepto de stakeholder, cabe considerar que la información podría ser solo para la entidad financiadora y no para los dueños del problema. Mucho más, si tenemos en cuenta el fomento de la colaboración universidad-empresa y la generación en las universidades de parques tecnológicos y spin-off que hacen del conocimiento un producto de mercado. Como investigadores, debemos tener en cuenta la tensión entre dueños del problema y empresa, con el fin de que la investigación responda a los interesados y no se convierta en un análisis de las necesidades de un grupo para generar productos que aquellos estén dispuestos a comprar. En este caso, la investigación estaría alejada de la justicia social y más cerca de un estudio de mercado.

La devolución de los resultados a los participantes puede que no provoque una mejora de la situación por problemas con el lenguaje o por no saber cómo abordar los cambios propuestos. El lenguaje de los académicos y la forma de publicar los resultados puede complicar la lectura, incluso si se hacen informes para los participantes (Kaltmeier, 2012). Es tradicional -e incluso obligatorio para algunos comités- que los participantes aprueben el informe final; sin embargo, hemos de plantearnos si nuestra escritura y forma de expresarnos es apropiada. La universidad tradicional empleaba la figura de la extensión como una forma de tener presencia y de relacionarse con la sociedad, lo que implicaba concebirla como algo diferente de la investigación. Pero aunque los resultados los entienda el grupo, puede suceder que no sea capaz de abordar las transformaciones que se recomienden. Greenwood (2000), expone cómo una de sus investigaciones surge de la solicitud de ayuda para abordar los cambios que solicitaba la investigación. Esto conlleva un compromiso por cambiar la realidad y no quedarse en una explicación, interpretación o predicción. Implica también, un compromiso con la realidad investigada y no solo tomar datos y desaparecer como seres atemporales (Barba et al., 2014).

\section{Cómo gestionar las tensiones entre el sistema y la investigación cualitativa}

Bourdieu (2003), considera que la clave para desenvolverse con solvencia en el campo en el que nos movemos, es conocer las reglas del juego y aprovecharlas. En este sentido, el modelo neoliberal ha conseguido difuminarlas y hacernos creer que las consisten en "publicar o morir" y en caso de aceptarlo, en "publicar hasta morir". Este lema encierra tras de sí la aceptación de una actividad frenética que no nos deja tiempo para reflexionar sobre las razones profundas de lo que hacemos y nos centra en aspectos meramente técnicos. Nuestro propósito es poner de manifiesto cómo, a través de la irreflexión sobre las reglas del juego, podemos abandonar los principios de la investigación cualitativa de forma sutil y dejarnos llevar por el sistema. No podemos olvidar que una de las bases del sistema neoliberal es considerar que las cosas son como son y no hay otra opción, lo que entraña que no es necesario reflexionar sobre lo que hacemos, sino que con el mero activismo es más que suficiente.

Nuestra propuesta plantea tomar distancia de la investigación y entender que reflexionar en la investigación es diferente de reflexionar sobre la investigación. Zeichner y Liston (1996) en la investigación docente, diferencian entre la reflexión en, que permite resolver los problemas que nos surgen, de la reflexión sobre, que nos faculta para comprobar en qué medida estamos cumpliendo nuestros propósitos y cómo podemos acercarnos a ellos. Nuestro cuestionamiento es una reflexión sobre la investigación, tratando de tomar distancia y observando los mecanismos que nos domestican -evidencias, estándares de investigación y cultura de las auditorías- y las posibilidades que tenemos para reflexionar sobre ellos. Entendemos que por el mismo sentido profundo de la investigación cualitativa no puede haber recetas universales sobre cómo enfrentarse a la domesticación y han de ser los procesos reflexivos de los investigadores los que resuelvan la tensión entre los requerimientos del sistema y los principios de la investigación cualitativa, sin olvidar que puede haber formas variadas de tomar conciencia (González Calvo \& Barba, 2014). Pero la reflexión no solo ha de ser individual, sino que debemos aprovechar los espacios que se nos ofrecen, como congresos, monográficos, revistas; etc. o crear otros nuevos para continuar el debate y asumir el 
reto de reinventarnos para que cambie la tendencia y la investigación cualitativa recupere su espacio.

El debate sobre el futuro de la investigación cualitativa se encuentra abierto. Comencemos por plantear cómo empoderarla por parte de los propios investigadores. St. Pierre (2011) considera que la investigación cualitativa, como en sus comienzos, ha de acercarse a la filosofía. Por su parte, Dimitriadis (2014) estima que hemos de volver la mirada a los años noventa, cuando los debates sobre la investigación cualitativa tomaban espacio a través iniciativas novedosas. Pero quizás la clave esté en las palabras de Torrance (2011), si se tiene en cuenta que la superación de la crisis de la investigación cualitativa ha de conllevar nuevas formas de ciencia y de gobierno, si bien, Lather (2014) observa que responder a la política de las auditorías ha de hacerse con unas prácticas que todavía no existen.

\section{Referencias}

Barba, J. J. (2013). La investigación cualitativa en educación en los comienzos del siglo XXI. En M. Díaz, \& A. Giráldez Hayes (Coords.), Investigación cualitativa en educación musical (pp. 23-38). Barcelona: Graó.

Barba, J. J., González-Calvo, G., \& Barba-Martín, R. A. (2014). Qué la fuerza esté contigo: Desvelar el lado oscuro de la investigación en educación. Magis: Revista Internacional de Investigación en Educación, 7(14), 125-140.

Barnett, R. (2001). Los límites de la competencia. El conocimiento, la educación superior y la sociedad. Barcelona: Gedisa.

Baudrillard, J. (1981). De la seducción. Madrid: Cátedra.

Bourdieu, P. (1997). Razones prácticas. Sobre la teoría de la acción. Barcelona: Anagrama.

Bourdieu, P. (2003). Cuestiones de sociología. Madrid: Itsmo.

Bruner, J. (2012). What psychology should study. International Journal of Educational Psychology, 1(1), 5-13. doi:10.4471/ ijep.2012.01

Carducci, R., Pasque, P. A., Kuntz, A. M., \& ContrerasMcGavin, M. (2013). Disrupting façades of clarity in the teaching and learning of qualitative research. Qualitative Research in Education, 2(1), 1-26. doi:104471/qre.2013.01

Creswell, J. W. (2011). Controversies in mixed methods. En N. K. Denzin, \& Y. S. Lincoln (Eds.), The Sage handbook of qualitative research (pp. 266-283. $4^{\mathrm{a}} \mathrm{ed}$.). Thousand Oaks, CA: Sage.

Denzin, N. K. (2009). The elephant in the living room: Or extending the conversation about the politics of evidence. Qualitative Research, 9(2), 139-160. doi:10.1177/1468794108098034
Denzin, N. K. (2010a). On elephants and gold standards. Qualitative Research, 10(2), 296-272. doi:10.1177/1468794109357367

Denzin, N. K. (2010b). The qualitative manifesto: A call to arms. Walnut Creek, CA: Left Coast Press.

Denzin, N. K. (2011). The politics of evidence. En N. K. Denzin, \& Y. S. Lincoln (Eds.), The Sage handbook of qualitative research (pp. 645-657. $4^{\mathrm{a}}$ ed.). Thousand Oaks, CA: Sage.

Denzin, N. K., \& Lincoln, Y. S. (2011). The discipline a practice of qualitative research. En N. K. Denzin, \& Y. S. Lincoln (Eds.), The Sage handbook of qualitative research (pp. 1-19. $4^{\mathrm{a}}$ ed.). Thousand Oaks, CA: SAGE.

Dimitriadis, G. (2014). The pedagogical turn: QI at 20 years. Qualitative Inquiry, 1-3. doi:10.1177/1077800414555068

Ellis, C. (2004). The ethnographic I: A methodological novel about autoethnography. Walnut Creek, CA: AltaMira Press.

Flick, U. (2015). El diseño de investigación cualitativa. Madrid: Morata.

Freeman, R. E. (1984). Strategic management: A stakeholder approach. Boston, MA: Pitman.

Freire, P. (2007). Pedagogía del oprimido. Madrid: Siglo XXI.

Fromm, E. (2006). El miedo a la libertad. Barcelona: Paidós.

Giroux, S., \& Tremblay, G. (2004). Metodología de las ciencias humanas. México D.F.: Fondo de Cultura Económica.

González-Calvo, G., \& Barba, J. J. (2014). Formación permanente y desarrollo de la identidad reflexiva del profesorado desde las perspectivas grupal e individual. Profesorado. Revista de Currículum y Formación del Profesorado, 18(3), 397-412.

Greenwood, D. J. (2000). De la observación participante a la investigación-acción participativa: Una visión crítica de las prácticas antropológicas. Revista de Antropología Social, 9, 27-49.

Greenwood, D. J. (2012). Doing and learning action research in the neo-liberal world of contemporary higher education. Action Research, 10(2), 115-132. doi:10.1177/1476750312443573

Guba, E. G., Lynham, S. A., \& Lincoln, Y. S. (2011). Paradigmatic controversies, contradictions, and emerging confluences, revisited. En N. K. Denzin, \& Y. S. Lincoln (Eds.), The Sage handbook of qualitative research (pp. 97-128. 4a ed.). Thousand Oaks, CA: Sage.

Hargreaves, A., \& Shirley, D. (2012). La cuarta vía. El futuro prometedor del cambio educativo. Barcelona: Octaedro.

Jemielniak, D. (2006). The management science as a practical field: In support of action research. The International Journal of Knowledge, Culture \& Change Managment, 6(3), 163-170. 
Jemielniak, D., \& Greenwood, D. J. (2015). Wake up or perish: No-liberalism, the social sciences, and salvaging the public university. Cultural Studies - Critical Methodologies, 15(1), 71-82. doi:10.1177/1532708613516430

Kaltmeier, O. (2012). Hacia la descolonización de las metodologías: Reciprocidad, horizontalidad y poder. En S. CoronaBerkin, \& O. Kaltmeier (Eds.), En diálogo: metodologías horizontales en ciencias sociales (pp. 25-54). Barcelona: Gedisa.

Kuhn, T. S. (2004). La estructura de las revoluciones cientificas. Argentina: Fondo de Cultura Económica.

Lather, P. (2006). Foucauldian scientificity: Rethinking the nexus of qualitative research and educational policy analysis. International Journal of Qualitative Studies in Education, 19(6), 759-772. doi:10.1080/09 518390600976006

Lather, P. (2012). The ruins of neo-liberalism and the construction of a new (scientific) subjectivity. Cultural Studies of Scientific Education, 7, 1021-1025. doi:10.1007/s11422012-9465-4

Lather, P. (2014). To give good science: Doing qualitative research afterward. Educational Policy Analysis Archives, 22(10), 1-14. doi:10.14507/epaa.v22n10.2014

Lawrence, P. A. (2008). Lost in publication: How measurement harms science. Ethics in Science and Environmental Politics, 8, 9-11. doi:10.3354/esep00079

Lincoln, Y. S. (2012). Los comités de conducta ética y el conservadurismo metodológico. Los cuestionamientos del paradigma fenomenológico. En N. K. Denzin, \& Y. S. Lincoln (Eds.), Manual de investigación cualitativa (pp. 332-363). Barcelona: Gedisa.

Morse, J. M. (2006). The politics of evidence. Qualitative Health Research, 16(3), 395-404. doi:10.1177/1049732305285482

Potter, J. (1996). Representing reality. Discourse, rhetoric and social construction. London: Sage.

Quindós, G. (2009). Confundiendo al confuso: Reflexiones sobre el factor de impacto, el índice h(irsch), el valor Q y otros cofactores que influyen en la felicidad del investigador. Revista Iberoamericana de Micología, 26(2), 97-102. doi:10.1016/S1130-1406(09)70018-X
Rivera-García, E. (2012). Reflexionando en torno a la investigación educativa. Una mirada crítica desde la auto etnografía de un docente. Qualitative Research in Education, 1(1), 36-57. doi:10.4471/qre.2012.03

Rodríguez-Romero, M. (2015). El paisaje amenazante de la producción de conocimiento científico en educación bajo el capitalismo cognitivo. Revista Interuniversitaria de Formación del Profesorado, 82(29.1). Recuperado de http:// goo.gl/YhU1YS

Shamir, R. (2008). The age of responsibilization: On market-driven morality. Economy and Society, 37, 1-19. doi:10.1080/03085140701760833.

Sparkes, A. C. (2013). Qualitative research in sport, exercise and health in the era of neoliberalism, audit and new public management: Understanding the conditions for the (im) possibilities of a new paradigm dialogue. Qualitative Research in Sports, Exercise and Health, 5(3), 440-459. doi:10.1080/ 2159676X.2013.796493

Spry, T. (2001). Performing autoethnography: An embodied methodological praxis. Qualitative Inquiry, 7(6), 706-732. doi:10.1177/107780040100700605

Pierre, E. A. (2011). Post qualitative research: The critique and the coming after. En N. K. Denzin \& Y. S. Lincoln (Eds.), The Sage handbook of qualitative research (pp. 627-643. 4a ed.). Thousand Oaks, CA: Sage.

Torrance, H. (2011). Qualitative research, science, and government: Evidence, criteria policy and politics. En N. K. Denzin \& Y. S. Lincoln (Eds.), The Sage handbook of qualitative research (pp. 569-580. 4a ed.). Thousand Oaks, CA: Sage.

Vasilachis de Gialdino, I. (2009). Los fundamentos ontológicos y epistemológicos de la investigación cualitativa. Forum Qualitative Sozialforschung / Forum: Qualitative Social Research, 10(2). Recuperado de http://goo.gl/w0AZl8

Villasante, T. R., \& Montañés, M. (2000). Algunos cambios de enfoque en ciencias sociales. En T. R. Villasante, M. Montańes \& J. Martí (Eds.), La investigación social participativa (pp. 13-28). Madrid: El Viejo Topo.

Zeichner, K. M., \& Liston, D. P. (1996). Reflective teaching. An introduction. Mahwah, NJ: Lawrence Erlbaum Associates Inc. Publishers. 